The clinical and biochemical findings are, however, not those generally associated with osteomalacia. The plasma calcium, phosphorus and alkaline phosphatase were all normal. Urinary calcium estimations were normal and calcium and phosphorus balance was strongly positive. Furthermore, no Looser's zones were identifiable in the skeleton and the disorder had a prolonged clinical course with failure of response to vitamin $D$ at doses varying from 1500 to 20,000 units/day.

Axial osteomalacia is difficult to distinguish from fibrogenesis imperfecta ossium on radiological grounds alone. Radiologically axial osteomalacia is characterized by a coarsening and distension of the trabecular pattern of the cervical vertebrae, lumbar vertebrae, ribs, and pelvis. (Figs. 1-4.) The more peripheral parts of the skeleton appear radiologically normal, unlike fibrogenesis imperfecta where they may not infrequently be involved. Histological examination of bone tissue from patients with fibrogenesis imperfecta ossium show a deficiency of collagen fibres as detected by the absence of birefringence with the polarizing microscope. There was, however, no histological evidence to suggest a diagnosis of fibrogenesis imperfecta ossium in the patient we have described. Urinary mucopolysaccharide and urinary hydroxyproline estimations were normal.
The prognosis of patients with 'axial osteomalacia appears to be reasonably good. One patient followed up for 5 years is still well without treatment (Arnstein $\frac{3}{\Phi}$ et al., 1967) and the symptoms and radiologicalo

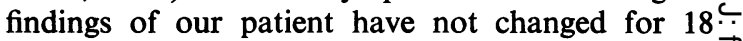
years.

Therapy with vitamin D, 20,000 units daily for 3 years has not induced any detectable alteration in $\frac{}{\sigma}$ bone biopsy histology and this fact together with $\overline{\bar{\omega}}$. the normal plasma biochemistry and positive calcium $\vec{\Phi}$ and phosphorus balance data strongly suggest that the disorder is not a true osteomalacia.

\section{Acknowledgments}

We thank Dr Paul Byers for reporting the bone biopsy histology and Dr E. H. Allen for radiological studies.

\section{References}

Arnstein, A. R., Frame, B. \& Frost, H. (1967) Recent progress in osteomalacia and rickets. Annals of Internal $\stackrel{\sim}{A}$ Medicine, 67, 1296.

Baker, S.L., Dent, C.E., Friedman, M. \& Watson, L. (1966) Fibrogenesis imperfecta ossium. Journal of Bome and Joint Surgery, 48, 804.

Frame, B., Frost, H.M., Ormand, R.S. \& Hunter, R. (1961) Atypical osteomalacia involving the axial skeleto Annals of Internal Medicine, 55, 632.

\title{
Secondary amyloidosis in relation to carcinoma of the kidney
}

\author{
DAVID SPENCER* \\ M.B., Ch.B., M.R.C.Path.
}

Senior Registrar, Area Department of Pathology, Exeter

WITH the reduction in the incidence and duration of chronic inflammatory diseases such as tuberculosis and chronic osteomyelitis during the past 2 decades, the pattern of aetiological factors in the causation of secondary amyloidosis has been changing. As a consequence other conditions are assuming more importance in the pathogenesis of this disease, notably, of course, rheumatoid arthritis.

This case report illustrates another of the now relatively more important causes, carcinoma of the kidney, which produced a sufficiently severe degree of secondary amyloidosis for the patient to develop renal failure.

\footnotetext{
* Present address: Department of Pathology, Wycombe General H ospital, High Wycombe, Bucks.
}

\section{Case report}

The patient was aged 59 at the time of his death in early 1969. He had been in Exeter on several occasions for minor ailments, the last of these being $\delta$ a Trendelenburg operation for varicose veins in $३$ August 1967. At this time the only other abnormality 윽 noted was that he also suffered from haemorrhoids, $\rightarrow$ but sigmoidoscopy was normal. The haemorrhoids thrombosed and settled down in May 1968.

However, his general practitioner, who saw him $ᄋ$ at this time, was not satisfied with his general con- $\mathbb{N}$ dition, mainly because of a weight loss of over a stone in a few months, with considerable hunger 0 despite eating well. As he also looked ill he was re- $c$ admitted for investigation in early June 1968.

On admission there was a pyrexia of $102^{\circ} \mathrm{F}$, a $\stackrel{\mathscr{C}}{+}$ 
palpable (three finger) liver, a palpable mass in the left hypochondrium (either spleen or kidney) and an enlarged left axillary lymph node.

Investigations at this time showed a haemoglobin level of $10.4 \mathrm{~g} / 100 \mathrm{ml}$, WBC normal, ESR $39 \mathrm{~mm} / \mathrm{hr}$, urine normal. Liver function tests showed a slightly raised bilirubin $(1 \mathrm{mg} / 100 \mathrm{ml})$ but the other results were within normal limits.

The enlarged axillary lymph node was biopsied, but showed no significant abnormality, and accordingly the mass in the left hypochondrium was investigated from the point of view of gastrointestinal or renal neoplasm. A barium enema was negative, but an intravenous pyelogram showed an enlarged lower pole of the left kidney, with little function and an abnormal calyceal pattern. No gross lesion could be seen in the right kidney, but there was poor renal excretion. The blood urea at this time was only $28 \mathrm{mg} / 100 \mathrm{ml}$.

A retrograde pyelogram showed the deformity in the left kidney. Laparotomy on 4 July 1968, confirmed the presence of a tumour in the left kidney and nephrectomy was performed. Renal vein involvement was found, with a $10-\mathrm{cm}$ string of tumour being taken from here and the adjacent vena cava. The excised kidney showed a tumour of $10 \mathrm{~cm}$ diameter which extended to but did not appear to breach the capsule and involved both the renal pelvis and the renal vein. Microscopically it was a typical renal carcinoma, with both tubular and clear cell components, and prominent vascular infiltration.

Following nephrectomy the patient had an uneventful convalescence; his pyrexia subsided and his weight increased.

He was re-admitted to hospital on 13 October, 1968, with palpitations and dizziness. At this time, apart from a slight pallor no abnormalities could be detected on examination, and in particular no masses could be felt in his abdomen. $\mathrm{Hb} 11.2 \mathrm{~g} / 100 \mathrm{ml}$, his ESR $28 \mathrm{~mm} / \mathrm{hr}$ and apart from some mild increase in vascular markings his chest $\mathrm{X}$-ray was normal. The attack did not persist, and he was discharged 4 days later to be re-admitted on 9 November with a further similar attack. On this occasion his pulse rate was $180 / \mathrm{min}$, BP $90 / 60 \mathrm{mmHg}$. He was treated with digoxin and quinidine and the tachycardia settled.

However, during this stay in hospital a second mass in the left hypochondrium was noted, thought both clinically and radiologically to be spleen.

Investigations. $\mathrm{Hb} 10.4 \mathrm{~g} / 100 \mathrm{ml}$, ESR of $51 \mathrm{~mm} /$ $\mathrm{hr}$, bilirubin $1.4 \mathrm{mg} / 100 \mathrm{ml}$ (of which $1.2 \mathrm{mg}$ was conjugated), alkaline phosphatase of $141 \mathrm{KA}$ units/ $100 \mathrm{ml}$, mainly of hepatic origin, and significantly his blood urea level had risen to $80 \mathrm{mg} / 100 \mathrm{ml}$. All other tests were normal. In view of the abnormal liver function tests a laparoscopy was performed, when the liver was seen to be rather swollen with a rounded edge. The surface was rather granular with some mottling but not typically cirrhotic. The spleen could not be visualized. A liver biopsy was performed which showed an essentially normal architecture, but a number of fairly sharply defined granulomata, of a tuberculoid type, were seen. Stains for tubercle bacilli were negative, and the differential diagnosis included sarcoidosis, a sarcoid-like reaction to adjacent tumour tissue and tuberculosis.

In view of a further rise in blood urea to $132 \mathrm{mg} /$ $100 \mathrm{ml}$ by 3 December with a seriously impaired creatinine clearance, amyloid studies were performed on the tissue available, using Congo Red with polarized light. Moderate amyloid deposits were found in the non-neoplastic area of the resected kidney and smaller deposits were found in the sarcoid-like lesions in the liver. The axillary lymph node originally excised was negative. The patient was therefore diagnosed as suffering from secondary amyloidosis due to carcinoma of the kidney.

His condition progressively deteriorated and he was finally re-admitted to hospital on 6 January, 1969 , with nausea, abdominal pain, vomiting and weakness. He showed a marked hepatosplenomegaly. His blood urea rose progressively $(208 \mathrm{mg} / 100 \mathrm{ml}$ on 9 January, $285 \mathrm{mg} / 100 \mathrm{ml}$ on 15 January); his urine output dropped to $220 \mathrm{ml}$ on 29 January, he passed into uraemic coma, developed bronchopneumonia and died on 31 January, 1969.

Necropsy. There were small multiple metastases in both lungs with bronchopneumonia. The liver was mildly enlarged $(1800 \mathrm{~g})$ being a little congested, but not granular. The spleen was moderately enlarged (475 g), being firm, with a waxy appearance, and the right kidney had a similar appearance, weighing $250 \mathrm{~g}$, both giving a strongly positive macroscopic staining with iodine. The renal vein stump and inferior vena cava were free of tumour, but there was a small recurrence in the bed of the left kidney.

Histologically massive amyloid deposits were confirmed in both spleen and the remaining kidney. The liver shows a marked reduction in the sarcoid-like reaction seen in the biopsy, but an increase in the amount of amyloid present. The adrenals were free of amyloid as were the lymph nodes. The heart was normal both macroscopically and histologically, and no aetiological factors for his attacks of palpitations could be found.

No chronic inflammatory foci or other causes of secondary amyloidosis were found at necropsy.

\section{Discussion}

The occurrence of secondary amyloidosis in relation to carcinoma of the kidney has been known since the time of Grawitz, who recorded the first case in 1883. Despite this, however, the comprehensive survey of this condition, by Ask Upmark in 
1940 , revealed a total of only sixteen recorded cases, to which he added two of his own. Further cases were recorded by Ellenburg (1943), Hayman \& Leiter (1946) and Kimball (1961).

The most recent review, by Azzopardi \& Lehner (1966), showed a total of twenty-six cases in the literature, to which they added two of their own.

This figure is surprisingly low, particularly as Azzopardi \& Lehner found in their series that $3.2 \%$ of the patients with renal carcinoma had secondary amyloidosis.

Three possible modes of pathogenesis of this association have been postulated. The first is that necrosis within a fairly slow-growing tumour may act as a chronic inflammatory focus. It could therefore be suggested that renal carcinoma, which often grows slowly, and is particularly prone to massive necrosis, satisfies these criteria, and hence the incidence is higher in this tumour than in other carcinomata. The second possibility is that the patient may have a concomitant chronic inflammatory disease with a coincidental carcinoma. However, Azzopardi \& Lehner's figures are against this. The third pathogenetic hypothesis is that there is a direct causal relationship between tumour and amyloid, without mediation by chronic inflammation.

The case under discussion is of particular interest in view of the hypotheses concerning pathogenesis. As regards necrosis within the tumour being a major factor, this is extremely unlikely in this case, principally because of the relationship of time to the course of the disease. The degree of amyloidosis suffered by this patient, judged by decreasing urinary function, by increasing amounts of amyloid present in biopsies and necropsy, and clinically by the increasing size of the spleen, became much more severe following nephrectomy. However, although the resected kidney showed necrosis, the tumour deposits found at necropsy were small and showed no necrosis. If necrosis were a major factor in causing amyloidosis severe enough to lead to death in $8 \frac{0}{0}$ months, then it would surely be present, after neph- $\underline{a}$ rectomy, in the metastatic lesions.

The second point is that the diagnosis of second- $\frac{D}{2}$ ary amyloidosis was made in life by reviewing the histology in light of the clinical progress. However, in view of the figures obtained by Azzopardi \& Lehner it is probably worth examining the non- 듬 malignant parts of kidneys removed for carcinoma by special stains for amyloid. This is certainly $\mathbb{\nabla}$ worthy of serious consideration as a diagnosis in a patient with failing renal function following removal ${ }^{\text {en }}$ of a carcinoma of kidney, particularly regarding. prognosis.

\section{Acknowledgments}

My thanks are due to Mr K. D. J. Vowles for permission to quote the case history and to $\operatorname{Dr} R$. A. Caldwell for his help and advice.

\section{References}

AsK UPMARK, E. (1940) On Amyloidosis induced by tumours N of the kidney. Acta medica Scandinavica, 54, 512.

Azzopardi, J.G. \& LeHNER, T. (1966) Systemic amyloidosid 윽 and malignant disease. Journal of Clinical Pathology, 1ब, 539.

Ellenburg, M. (1943) Amyloidosis secondary to maligna Grawitz tumour (hypernephroma) of the kidnes Journal of the Mount Sinai Hospital, 10, 323.

GrawITZ, P (1883) Virchows Archiv für pathologische Anatomie und Physiologie, 93, 39. Quoted by Ask Upmad (1940).

Hyman, A. \& Leiter, H.E. (1946) The association of hype nephroma with amyloidosis of the kidney. Journal Urology, 56, 303.

Kimball, K.G. (1961) Amyloidosis in association with neoplastic disease. Annals of Internal Medicine. 55, 958.

\section{Addendum}

Following preparation of this report a second case $\overrightarrow{\vec{F}}$ was found in which moderate amyloid deposits were $\frac{0}{3}$ demonstrated in the resected kidney. The patient has not yet shown evidence of impaired renal function. 